\title{
The Application of Cooperative Diversity in the CDMA System Based on Relay Communication
}

\author{
Hancheng Liao \\ School of Software and Communication Engineering \\ Jiangxi University of Finance and Economics \\ Nanchang, China, 330013 \\ Liaoemail@163.com
}

Keywords: Cooperative Diversity, CDMA, Relay

\begin{abstract}
The cooperative diversity technology in code division multiple access system is studied in this paper. Some mobile terminals relay signals for each other so as to achieve the spatial diversity gain. The cooperative scheme in the mobile terminal is discussed. The uplink system model, as well as the receiving structure is given. The performance advantage of such system is clearly indicated through computer simulation.
\end{abstract}

\section{Introduction}

Code division multiple access system is famous for combating multipath fading in wireless communication systems [1]. Cooperative diversity is a cooperative multiple antenna technique for improving performance or channel capacities which exploits user diversity by decoding the combined signal of the relayed signal and the direct signal in wireless networks [2-4]. A conventional single hop system uses direct transmission where a receiver decodes the information only based on the direct signal while regarding the relayed signal as interference, whereas the cooperative diversity considers the other signal as contribution. Hence, cooperative diversity is considered as an antenna diversity method that uses distributed antennas belonging to each node in wireless networks. The uplink CDMA system model of LAS spread-spectrum communication system using such cooperative diversity technology is studied as follows [5-6].

\section{System Model}

Practically, more than one antenna can be installed on the base station (BS) for a CDMA mobile communication system, but only one antenna can be installed on each mobile terminal (MT) because of its limited size. For simplicity, we suppose that the number of antenna in the mobile terminal and in the base station is both only one. Although both transmitting operation and receiving operation can make use of the same antenna by time-division duplex (TDD), we can assume that two antennas are mounted-one is focusing on transmitting data and the other is focusing on receiving data. Furthermore, we take it for granted that perfect power control technology is implemented to completely eliminate the near-far effect in all cells. As for the multiple access codes, LAS codes are employed [6-7]. In addition, it is a relay technology in nature when we refer to cooperative diversity.

In this paper, we only talk about a single cell. Naturally, there are many active mobile terminals in each cell for such mobile system. Suppose that the base station has chosen K mobile terminals as a group for some short period by a given rule-being in near area, having similar angle-of-arrival (AOA), indicating the same distance to the base station. During such period, they are cooperators, that is to say, every one of them is be in duty bound to relay the data from the others. We only discuss the situation that the relay number of times is one and the links with twice relays are not taken into consideration. Such cooperative CDMA cell is illustrated in Fig. 1. In this situation, one base station is placed in the center and one omnidirectional antenna is installed on it. 

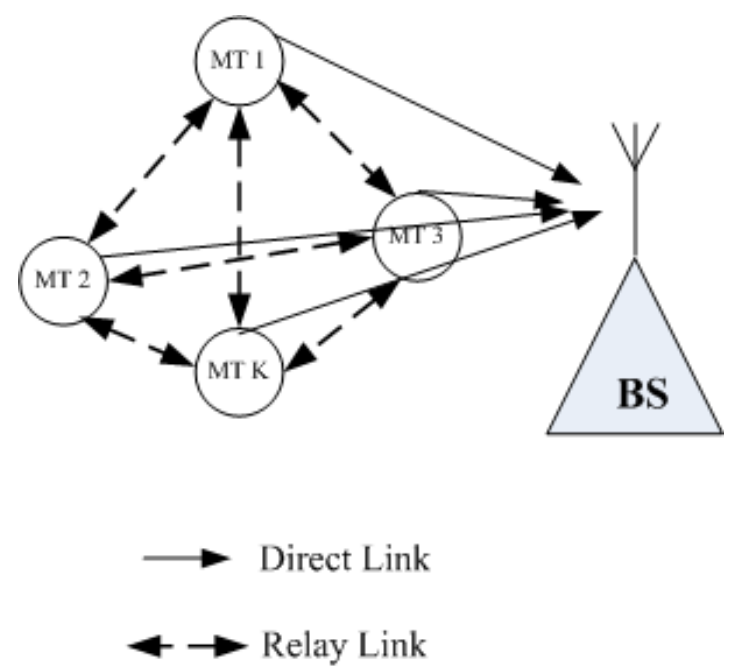

Figure 1. A cooperative CDMA cell with K cooperative mobile terminals and one base station

As we know, every each mobile terminal can acquire the spread-spectrum sequence for each other from the base station. Here, we begin with only one group consisting of $\mathrm{K}$ members and each member has stored K MACs from the beginning of cooperation. Other cases of many groups in a cell with either equal or different number of members in a group can be studied in further work. Also, we don't take such situation into account that any cooperative mobile terminal is working irregularly, no matter because of power cutting, interrupt or breakdown. The allocation scheme of time slots (TSs) in such cooperative uplink system is shown in Fig. 2. During the first K time slots, each mobile terminal transmits its data successively, e.g., No. k mobile terminal (named MT k) sends at No. k time slot (named TS k). Meanwhile, the other K-1 mobile terminals and the base station are receiving such data. Then it is followed by K-1 relay time slots, in which each one mobile terminal transmit data of the others by a given order. As shown in Fig. 2, the data of the first mobile terminal is transmitted by MT $\mathrm{K}$ in TS $\mathrm{K}+1$, by MT K-1 in TS $\mathrm{K}+2$, up to by MT 2 in TS $2 \mathrm{~K}-1$. Although there are more time slots than systems without cooperation, the communication efficiency is not reduced obviously in general according to the fact that a lot of mobile terminals have spare time in many kinds of business or even they are in standby mode.

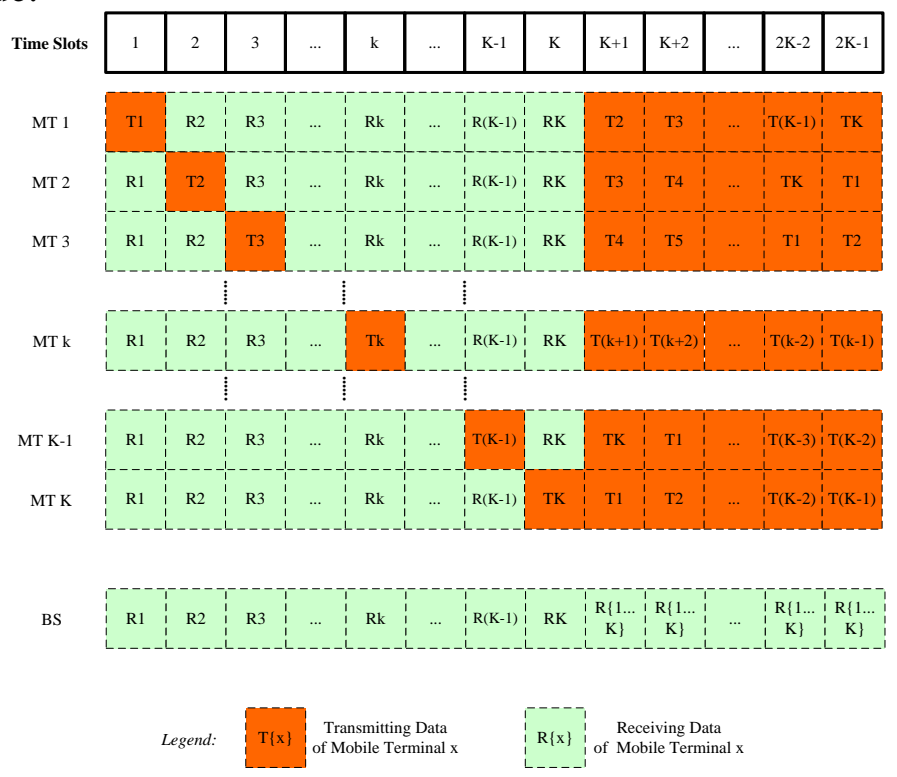

Figure 2. The allocation scheme of time slots in uplink system for K cooperative mobile terminals and one base station

Then we go on to the system model of any mobile terminal. Without loss of generality, we can only research the first mobile terminal. Its model is given in Fig. 3, where only baseband signals and operations are mentioned. The transmitting and receiving signal of MT $\mathrm{x}$ is notated as $s_{x}(t)$ and 
$r_{x}(t)$. During the first time slot (named TS 1), MT 1 transmits its data. During the next K-1 time slots, MT 1 receives data from MT 2 to MT K in turn. In the figure, $\hat{s}_{p}^{n}$ represents the despread symbol of MT n received by MT p using MAC $c_{n}$, it is an estimate of symbol $s_{n}^{n}$ for MT $\mathrm{n}$ and should be transmitted by MT p hereafter. The amplifying coefficient can be simplified as $a_{p}^{n}=1 /\left\|\hat{s}_{p}^{n}\right\|$ when PSK is employed. During the last K-1 time slots, the relay signals $a_{p}^{n} \hat{s}_{p}^{n}$ is spread with $c_{p}$ and transmitted by MT p separately.

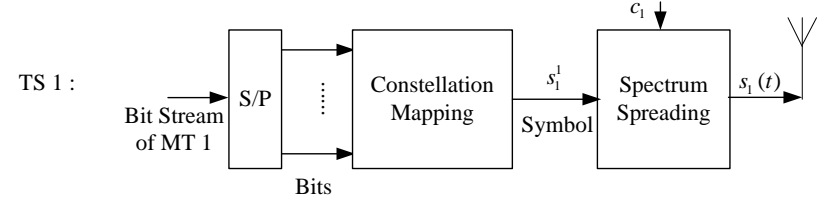

(a) Time slot 1

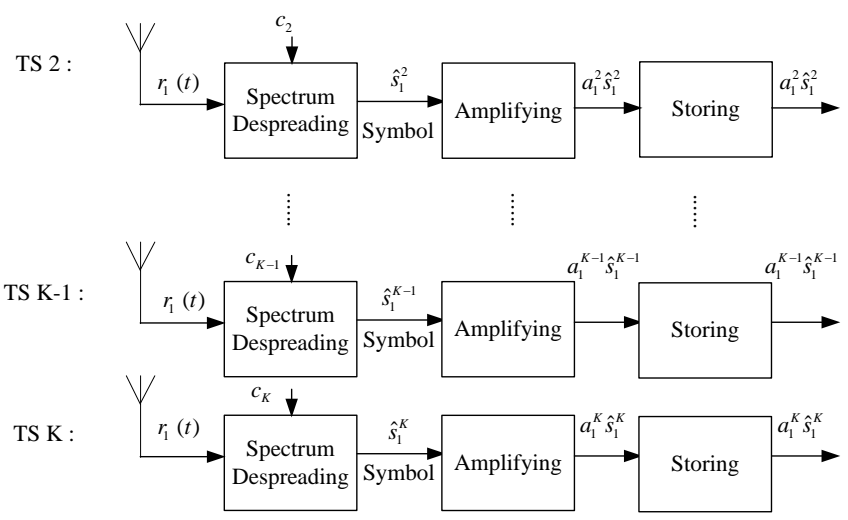

(b) Time slot from 2 to $\mathrm{K}$
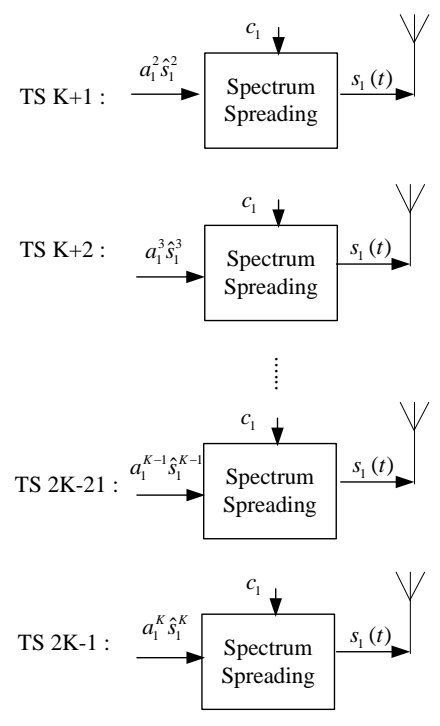

(c) Time slot from $\mathrm{K}+1$ to $2 \mathrm{~K}-1$

Figure 3. The uplink system model for the first cooperative mobile terminal (MT 1)

The corresponding uplink system model of base station is given in Fig. 4, where $r(t)$ is the received signal and $r_{j}^{n}$ is the despread symbol for MT n using MAC $c_{n}$ during TS $\mathrm{j} . r_{K+1}^{1}, r_{K+2}^{1}, \ldots$, $r_{2 K-1}^{1}$ and $r_{1}^{1}$ are prepared for the next diversity combining operation. As the modulation type is PSK, we can choose equal gain combining (EGC) method and let $r^{1}=\bar{r} /\|\bar{r}\|$, where $\bar{r}=\left(r_{1}^{1}+r_{K+1}^{1}+\cdots+r_{2 K-1}^{1}\right) / K$.

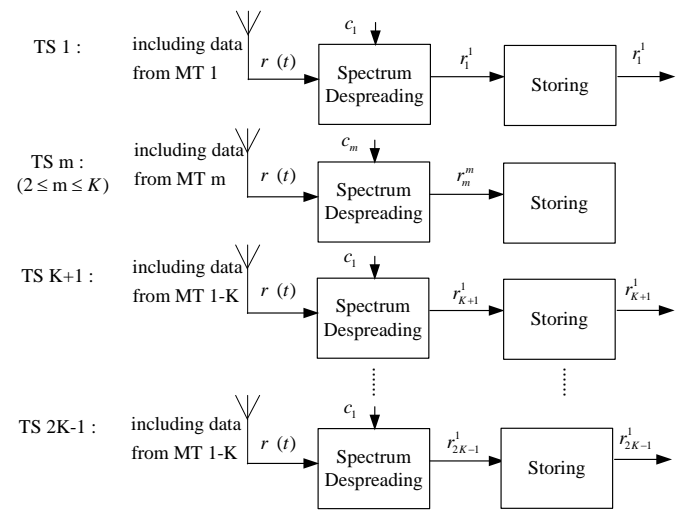

(a) Time slot from 1 to $2 \mathrm{~K}-1$

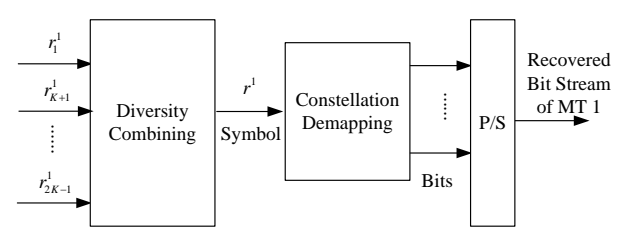

(b) After time slot 2K-1

Figure 4. The uplink system model for the base station (only showing the operation concerning MT 1) 


\section{Performance Simulation}

In the simulation, both system with cooperation $(\mathrm{K}>1)$ and system without cooperation $(\mathrm{K}=1)$ are considered. The spread factor is 16 and modulation type is QPSK. The channel model is ITU-VA 120 $\mathrm{km} / \mathrm{h}$. The base station knows the channel state information (CSI) but the mobile terminals don't. The bit error rate (BER) performance is shown in Fig. 5 . The diversity gain is obvious and almost linear to $\mathrm{K}$ when $\mathrm{K}$ is not very large.

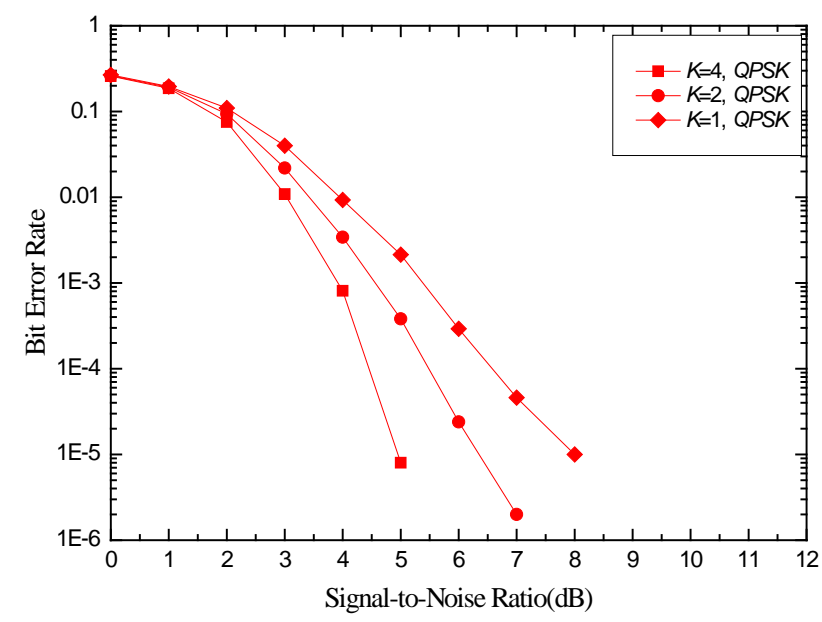

Figure 5. The BER performance of cooperative CDMA system

\section{Summary}

By applying cooperative diversity technology to existing CDMA spread-spectrum system, the system performance and capacity can be much better. As the number of cooperative users increases, the diversity gain increases too. Our numerical simulation reveals that the cooperative scheme is able to extract the full distributed spatial diversity in the uplink communication. Of course, there is trade-off between the diversity gain and the loss of other wireless resource such as time and power resources because they are wasted to relay the signal from the source node to the destination node. Therefore, it is noteworthy that the number of cooperative users is well chosen.

\section{References}

[1] A. Viterbi: CDMA: Principles of Spread Spectrum Communication, Addison Wesley, New York (1995)

[2] J. N. Laneman, D. Tse and G. W. Wornell:Cooperative Diversity in Wireless Networks: Efficient Protocols and Outage Behavior, IEEE Trans. Inf. Theory, Vol. 50, No. 12(2004), p.3062

[3] A. Bletsas, H. Shin and M. Z. Win: Cooperative Communications with outage-optimal opportunistic Relaying, IEEE Trans. Wireless Commun., Vol. 6, 9(2007), p.3450-3460

[4] MinChul Ju, Kyu-Sung Hwang and Hyoung-Kyu Song:Relay Selection of Cooperative Diversity Networks with Interference-Limited Destination, IEEE Trans. Veh. Technol., Vol. 62, No. 9(2013), p.4658-4665

[5] Hancheng Liao, Daoben Li and Qingrong Zhang: An example of LS codes, 16th International Conference on Computer Communication, Beijing, China (2003), p. 918

[6] Daoben Li: A spread spectrum coding method possessing interference free window, Chinese Patent PCT/CN00/0028 (1999)

[7]L. Hanzo, L. L. Yang, E. L. Kuan and K. Yen, Single and Multicarrier DS-CDMA, IEEE Press (2003) 